\title{
Meeting Report: PQRI Workshop on Application of IVIVC in Formulation Development
}

\author{
Vivian Gray \\ Dissolution Technologies, Hockessin, DE, USA
}

$\mathrm{T}$ he PQRI workshop, cosponsored by AAPS, FIP, USP, and FDA, was held in Bethesda, Maryland, USA, on

September 5 and 6, 2012. The Chairs of the meeting were Avraham Yacobi and Vinod Shah. The Scope and Objectives of the Workshop were as follows:

1) Review the status of In Vitro-In Vivo Correlations (IVIVC), discuss applications and potential benefits of IVIVC.

2) Review and evaluate different methodologies and their potential use in IVIVC assessment.

3) Assess advantages and limitations of IVIVC in formulation development.

4) Develop the basis for a summary paper on IVIVC and a publication as a PQRI document.

Session I: Foundation of and Advances in IVIVC

Avi Yacobi gave the Welcome Remarks. He reviewed the definitions and parameters of IVIVC and related the origins of the IVIVC. The Workshop was planned to be interactive with Panel discussions and Breakout Sessions. He discussed the constraints on developing an IVIVC and put forth some topics for consideration. He outlined the program for the next two days.

The first talk, "Traditional IVIVC Using Deconvolution: Advantages and Drawbacks," was given by Mario González from P'Kinetics International. He reviewed the development of the FDA Guidance, Extended Release Oral Dosage Forms: Development, Evaluation, and Application of In Vitro/In Vivo Correlations, and USP General Chapter $<1088>$ In Vitro and In Vivo Evaluation of Dosage Forms. USP $<1088>$ includes a case study that uses nonlinear correlations, and he emphasized that the correlation does not always have to be a straight line. He pointed out the problems with the Wagner-Nelson equation and reiterated that deconvolution is the best way to handle data for an IVIVC. The benefits of the IVIVC (e.g., reducing number of BA studies, supporting dissolution specifications, and use in biowaivers) were explained. Then Jennifer Dressman from University of Frankfurt gave a presentation titled "Review of GI Physiology and Use of Bio-relevant Dissolution Media." She began her talk with a hypothesis that the closer the dissolution test conditions are to the physiology, the better the chances of predicting in vivo performance. When developing the best dissolution test, there are three important considerations: (1) the section of the Gl tract where the drug is released from the dos- age form, (2) the time that the dosage form has to release the drug, and lastly (3) the composition of the fluids into which the drug is released. She discussed designing an appropriate dissolution test by considering the solubility and permeability of the drug and the dosage form. For highly soluble drugs, simple buffers should be sufficient. For less soluble drugs with high permeability, media that simulates the body fluid and meets sink conditions should be considered. If the drug is poorly soluble and has low permeability, using sink conditions may lead to overprediction of absorption. There were several case studies to illustrate these points. She summarized by stating that the best dissolution test to generate an IVIVC will consider the drug properties, mechanism of release, dosage form dimensions, excipient properties, and dosing conditions in the in vivo study. The next speaker was Theresa Shepard from the Medicines and Healthcare Products Regulatory Agency (MHRA); she discussed "EMA Guidance on MR Dosage Forms." She informed the audience that the EMA Guidance, Note for Guidance on Quality of Modified Release Products: A. Oral Dosage Forms, B. Transdermal Dosage Forms, Section I (Quality) and Section II on Pharmacokinetics and Clinical Evaluation, would be revised at some later date to include aspects of IVIVC. She related that the anticipated IVIVC topics that would be covered in the Quality section are as follows: definitions of levels of IVIVC, advantages and disadvantages of the different levels of IVIVC, the role of IVIVC and program rationale for formulation choice, reference formulations, and extensions for IVIVC. Additional topics in the Quality section would be the study design (dissolution media sampling times) and applications of specification setting and biowaivers for product variations. The IVIVC topics that would be in the Clinical section are in vivo study design, IVIVC analysis, validation, and reporting. She pointed out that the major reason for an IVIVC is that it provides in vitro release testing as a surrogate for the BE study, therefore saving money and time. One of the reasons the regulators encourage IVIVC is that with post-approval, there is the reassurance that a positive benefit/risk balance will be maintained throughout life of the product. She gave examples of case studies of the many possibilities of product variations. Dr. Shepard concluded that there are valuable opportunities from an IVIVC provided the dissolution test is discriminatory and provides a link to the clinical batches. 
After the break, Doug Smith of DFS Consulting spoke on "Applications of IVIVC in Formulation Development." He began his presentation by stating the key applications of IVIVC, for example, assisting in understanding key formulation variables, providing biowaivers for changes in the product, and setting dissolution specifications other than $\pm 10 \%$. The requirements of the In Vivo side of an IVIVC are to test three formulations with different release rates; compare formulations to a solution, immediate-release (IR), or intravenous (IV) reference; and perform a crossover study on fasted subjects. The In Vitro side requires formulations exhibiting at least a $10 \%$ dissolution rate difference, use of typical apparatus, adjustment of the dissolution method to fit the in vivo data, and use of time scaling when necessary. He presented five case studies with two on IR products and three on modified-release (MR) products. He defined external and internal validation and stated that proof of external validation provides greater confidence in IVIVC than internal validation. Traditional and nontraditional approaches to Level A IVIVC were described. He concluded with an explanation of the benefits of IVIVC: it provides a framework for formulation development, promotes prioritizing of formulation efforts, places development of a biorelevant dissolution method formally into the development process, defines manufacturing parameters at an early stage, and reduces the risk of requiring Phase III to market bioequivalence (BE) studies. The last speaker of the session was Tahseen Mirza of the FDA. His talk was "Use of IVIVC to Facilitate Product Development via 'Quality by Design' Approach." His objective was to show examples of how to integrate IVIVC and Quality by Design $(\mathrm{QbD})$ concepts into drug development. The real dilemma is how to determine if batches that fall within and outside of the design space have any clinical relevance. He suggests that we build in quality from the beginning during preclinical and Phase 1 . He described the Micro Dissolution Apparatus and its advantages, the principal one being that only small amounts of drug are needed for the test. He also posed the possibility of predicting dissolution and human exposure by near infrared (NIR) and presented a case study. He concluded that establishing QbD early in the candidate selection and drug development process along with more reliance on IVIVR could provide clinically relevant methods.

\section{Session II: New Methodologies Assessment- Simulation of IVIVC}

The session started with a talk titled "IVIVC Perspective, Generic Pharmaceuticals," given by Russell Rackley from Mylan Pharmaceuticals. He began with a definition of the term "biorelevant dissolution" as a system for developing extended-release products based upon the prediction or simulation of the entire plasma level-time curve expected from the administration of a controlled-release oral dosage form. It employs product in vitro dissolution behavior and drug pharmacokinetic parameters in conjunction with a classical pharmacokinetics (PK) model. He discussed the three ways simulation may be used in drug development: simulate plasma profiles based on release profiles and knowledge of PK, simulate plasma levels based on in vitro for correlation with in vivo data to support validation of IVIVC, and lastly, simulate the dissolution environment for correlation with in vivo. He discussed some assumptions for an ideal in vitro-in vivo relationship (IVIVR), in particular that (1) the in vitro release characterization is relevant to observations of absorption in vivo, (2) the formulation is robust to in vivo perturbations, (3) the drug substance has Biopharmaceutics Classification System (BCS) Class 1 characteristics, and (4) the IVIVR is applied to MR formulations with release rate limited absorption. He gave a case study using Lopressor Oros. The barriers to IVIVC are low solubility of the drug substance, food effects, and in vivo or in vitro variability. He gave a practical approach for IVIVC use in the generic industry, acknowledging that a validated IVIVC is not typically done, as the benefit versus investment is uncertain. However, IVIVR concepts should be investigated to support finetuning the formulation. Level A is rarely used, with Level $C$ IVIVC being the more common, based primarily on PK parameters for $\mathrm{BE}$.

The second speaker was Rong Li from Pfizer giving the innovator industry perspective for IVIVC. She pointed out that the primary objective for an IVIVC is to obtain biowaivers by using the dissolution test as a surrogate for pharmacokinetic data. She discussed the approaches undertaken to establish an IVIVC. These may include a retrospective analysis of existing PK/dissolution data along with the more typical prospective planning and developing of clinical study designs for IVIVC. She presented several case studies. The challenges when establishing an IVIVC were discussed. Some examples include obtaining multiple release rates while maintaining the same release mechanism, which is not trivial, and developing an IVIVC on BCS Class 2 immediate-release drug products. In addition, there is the question of whether there should be a standardized approach to evaluate dosage dumping. Michael Bolger, from Simulations Plus, then gave a talk titled "GastroPlus: Mechanistic Deconvolution and the Role of Physiological Modeling in IVIVC." He described IVVIC as a mathematical input function that allow us to use in vitro release to predict in vivo plasma concentration-time data. He went on to say that IVIVC could allow us to generate such a function to predict the plasma concentration-time for different formulations with different in vitro release time profiles. He described the use of GastroPlus for deconvolution with traditional methods, such as model-dependent and model-independent options. The drawbacks to using this traditional method, such as what are the output and limitations of assumptions, were reviewed. Another 
method for deconvolution in GastroPlus is a mechanistic absorption method. In this approach, additional inputs such as physiological parameters and drug properties are considered, and the output is a model that combines all available in silico, in vitro, and in vivo information. The determination continues by finding the correlation between the deconvoluted in vivo release and in vitro profiles through various means, and then predicting by convolution the plasma concentration-time profile. He concluded by relating the advantages of an IVIVC and how the GastroPlus mechanistic approach allows you to separate the in vivo dissolution of your formulation from absorption and first-pass extraction. The DDDPlus program that assists in designing hypothetical formulations and dissolution experimental conditions was mentioned.

Jason Chittenden from Pharsight/Centara ended the session with two talks. His first subject was a mechanistic case study for IVIVC using the Simcyp ADAM Model and metoprolol MR. Model 1 used functional numerical deconvolution with the oral solution as the reference. Model 2 used the Simcyp semimechanistic parent/metabolite model. He showed how a new once-daily formulation of metoprolol could be designed using Simcyp. He also discussed how Simcyp could help evaluate the performance of newly designed formulations in various disease groups, different ethnic groups, and the elderly and pediatric populations. The simulated PK profiles are linked to pharmacodynamics models to estimate and understand the efficacy of the newly designed formulation. He pointed out that mechanistic IVIVC models are useful for BCS Class 3/4 drugs as they distinguish dissolution, gastrointestinal ( $\mathrm{Gl}$ ) transit, and permeation processes and avoid confounding. His second talk was on the Phoenix IVIVC Toolkit. He described the Phoenix WinNonlin as a leading PK/PD (pharmacodynamics) data analysis tool. It is a separately licensed extension of WinNonlin functionality that provides a stand-alone tool using deconvolution (numerical, Wagner-Nelson, and Loo-Riegelman), convolution, and the Levy Plot. The Toolkit can be used to assist in designing formulations, evaluating the dissolution experiment, setting dissolution specifications, investigating absorption, and exploring correlation models for one-stage IVIVC.

Breakout Sessions followed Session II. The topics and moderators were as follows:

\section{Breakout Session A-Evolution of Traditional IVIVC and an Eye on QbD}

Moderators: Barbara Davit, FDA, and Mario González, P'Kinetics International

\section{Breakout Session B-Understanding the Role of Bio- relevant Dissolution Testing-It's Not Just About the Media}

Moderators: Jennifer Dressman, University of Frankfurt, and Erika Stippler, USP

\section{Breakout Session C-Role of Physiological Modeling in IVIVC}

Moderators: Michel Bolger, Simulations Plus, and Jason Chittenden, Pharsight

\section{Breakout Session D-Role of Population Approaches in IVIVC}

Moderators: John Duan, FDA, and Derek Ganes, Consultant

\section{Session III: Application of IVIVC}

Derek Ganes started the session with a talk titled "When is IVIVC Applicable?-A Practical View." He stated that IVIVC may be used in the development of generic MR formulations. He explained that an IVIVC requires that the in vitro dissolution method is predictive of the in vivo drug absorption. The IVIVC method is based on time scaling and modeling of the GI transit. He gave two examples of pilot studies that were conducted using slow-, medium-, and fast-release prototype formulations and referenced with fasting and fed subjects. The first steps were to use the Wagner-Nelson method of deconvolution to determine the amount of drug absorbed in vivo. He proceeded to describe simulations and modeling in each example to predict the amount of drug absorbed. The second talk was given by Jim Polli, University of Maryland, on "How Can IVIVC/IVIVR Be Used?" Dr. Polli discussed the different categories of the IVIVC/IVIVR. He rated the categories, giving a $A A A$ to the FDA level $A$ of convolution, a AA rating for deconvolution, A to linear (USP level A) deconvolution, $B$ to summary parameters, $C$ to point estimates, and $D$ to rank order. He outlined the reasons for an unsuccessful IVIVC: an inadequate IVIVR model, the in vitro dissolution was a quality control (QC) test that did not replicate in vivo performance, and in vivo study variability problems. He suggested that when IR dosage forms are not dissolution controlled, modest dissolution changes are of no consequence in vivo. He gave two IVIVR case studies. He concluded by stating that the in vitro dissolution test can elucidate factors controlling absorption kinetics and dosage form performance through the $f_{2}$ criteria, dissolution kinetics, and connection to Caco-2 permeability. The next speaker was Sandra Suarez-Sharp from the FDA who gave a talk titled "FDA's Experience on IVIVC-New Drugs." She showed statistics related to the use of IVIVC in NDA submissions. Over $69 \%$ of the IVIVC models were seen in oral dosage forms, $67 \%$ of these models employed the two-stage independent approach, and around $80 \%$ were ER formulations. She provided types of dissolution media used in the IVIVC: Simulated Gastric Fluid, Test Solution (TS) (without pepsin); $0.01 \mathrm{~N} \mathrm{HCl}$ with $0.05 \%$ sodium lauryl sulfate (SLS) and $0.7 \%$ sodium chloride; $0.04 \mathrm{M}$ sodium phosphate buffer $\mathrm{pH} 6.8$ with $2 \% \mathrm{SLS}$; water; ethanol/water (90/10); and a two-part system of $\mathrm{pH} 4.8$ citrate buffer adjusted to $\mathrm{pH} 6.6$ at $5 \mathrm{~h}$. Some reasons for unacceptable IVIVCs are no real difference in the dissolution 
rate, lack of rank order, and use of different scaling factors for the three formulations. Additionally, some other causes for IVIVC failure were that the IVIVC model did not meet the validation criteria, mean-based deconvolution was used instead of individual-based deconvolution, the model was not developed under fasted conditions, and the model was over-parameterized and not fully mechanistic. She concluded with several overall considerations for IVIVC: IVIVC can be possible for some IR formulations and novel dosage forms, FDA does not specify the kind of modeling approaches, and successful IVIVC models can be possible when simple dissolution methods are used. The last speaker of the session was Barbara Davit of FDA on the FDA experience on IVIVC-Generic Drugs. She first reviewed the FDA Guidance for Industry on IVIVC. She described the three common uses of IVIVC with the generics industry: Level 3 changes in scale-up and postapproval changes (SUPAC-MR) (manufacturing site, non-release controlling excipients, process, and releasecontrolling excipients), complete removal of non-release controlling excipients, and setting dissolution specifications. There were five case studies where the applicants' approaches and the FDA assessments were provided. She discussed that an IVIVR is not as robust as an IVIVC but can be an important feature in a $\mathrm{QbD}$ approach with respect to generic drug development. An IVIVR can be used as a prediction of commercial batch performance and assessment of post-approval changes. Additionally, other uses of IVIVR are to perform convolution of the in vitro data for reference and prototype generic formulations, to predict the outcome of BE studies using various prototype formulations, and to establish a linkage between exhibit and commercial batches. In summary, the FDA guidance provides recommendations for properly establishing IVIVC of generic modified-release oral drug products, and IVIVC can be used to support some post-approval changes and changes in dissolution methods and specifications. Very few submissions to the FDA Office of Generic Drugs contained IVIVC data, and most were not acceptable. The Office of Generic Drugs proposes using IVIVR as part of a $\mathrm{QbD}$ approach to the development of generic modifiedrelease formulations.
Breakout Sessions followed:

\section{Breakout Session E-Uses of IVIVC Data to Support Biowaiver}

Moderator: James Polli, University of Maryland, and Tahseen Mirza, FDA

\section{Breakout Session F-IVIVC and IVIVR for IR Dosage Forms}

Moderators: Maziar Kakhi, FDA, and Doug Smith, DFS Consulting

\section{Breakout Session G-Focus on MR Dosage Forms} Moderators: Mario González, P'Kinetics International, and John Duan, FDA

\section{Breakout Session $\mathrm{H}$-Untangling the Regulatory Knots-The Road Map to Harmonized Guidances Worldwide \\ Moderators: Theresa Shepard, MHRA, and Sandra Suarez-Sharp, FDA \\ The afternoon session consisted of summary reports from each of the Breakout Sessions, followed by a Q and A session.}

\section{Session IV: Where Do We Go From Here?}

Vinod Shah, filling in for Tony DeStefano of USP, wrapped up the workshop with a talk on "What Is the Future?" that reviewed the goals and objectives of the workshop. The concept of mapping, an idea comparable to IVIVC, was discussed. There is to be a PQRI Report with goals to stimulate firms to develop IVIVC/IVIVR for all types of dosage forms for branded as well as generic products, to generate research in the area of simulations to help design better products, and to stress that the regulatory authorities will support, welcome, and help in the new developments.

Avi Yacobi closed the meeting with remarks thanking the audience for its participation and praising the speakers and moderators for the excellent material presented. He further discussed the next steps and spoke of a possible global guidance for IVIVC. 